\title{
Heterogeneity and changes in preferences for dying at home: a systematic review
}

\author{
Barbara Gomes*, Natalia Calanzani, Marjolein Gysels, Sue Hall and Irene J Higginson
}

\begin{abstract}
Background: Home-based models of hospice and palliative care are promoted with the argument that most people prefer to die at home. We examined the heterogeneity in preferences for home death and explored, for the first time, changes of preference with illness progression.

Methods: We searched for studies on adult preferences for place of care at the end of life or place of death in MEDLINE (1966-2011), EMBASE (1980-2011), psycINFO (1967-2011), CINAHL (1982-2011), six palliative care journals (2006-11) and reference lists. Standard criteria were used to grade study quality and evidence strength. Scatter plots showed the percentage preferring home death amongst patients, lay caregivers and general public, by study quality, year, weighted by sample size.
\end{abstract}

Results: 210 studies reported preferences of just over 100,000 people from 33 countries, including 34,021 patients, 19,514 caregivers and 29,926 general public members. $68 \%$ of studies with quantitative data were of low quality; only 76 provided the question used to elicit preferences. There was moderate evidence that most people prefer a home death-this was found in $75 \%$ of studies, $9 / 14$ of those of high quality. Amongst the latter and excluding outliers, home preference estimates ranged $31 \%$ to $87 \%$ for patients ( 9 studies), $25 \%$ to $64 \%$ for caregivers (5 studies), $49 \%$ to $70 \%$ for the public (4 studies). $20 \%$ of 1395 patients in 10 studies (2 of high quality) changed their preference, but statistical significance was untested.

Conclusions: Controlling for methodological weaknesses, we found evidence that most people prefer to die at home. Around four fifths of patients did not change preference as their illness progressed. This supports focusing on home-based care for patients with advanced illness yet urges policy-makers to secure hospice and palliative care elsewhere for those who think differently or change their mind. Research must be clear on how preferences are elicited. There is an urgent need for studies examining change of preferences towards death.

Keywords: Patient preference, Hospice care, Palliative care, Hospices, Terminal care, Patient satisfaction

\section{Background}

Debates about the institutionalization of death and the fact that most people die in hospital have happened within and between countries since the beginning of the hospice movement [1-3]. The issue has gained momentum in light of national and international projections showing dramatic rises in numbers of deaths due to increased life expectancy and large cohorts of "baby-boomers" reaching older ages [4-6]. As chronic conditions are increasingly leading causes of death $[4,7]$, most people should expect a period of terminal illness to precede death. This urged projections of

\footnotetext{
* Correspondence: barbara.gomes@kcl.ac.uk

King's College London, Cicely Saunders Institute, Department of Palliative Care, Policy \& Rehabilitation, Bessemer Road, London SE5 9PJ, UK
}

place of death, with a view to inform how the care of terminally ill patients is planned for the future [8-10].

Many countries including the United States (US) and the United Kingdom (UK) have increased the focus on home-based models of hospice and palliative care with the argument that most people prefer to die at home. Trends of increasing home deaths have followed [11-14]. Indeed, a review of the cancer literature in 2000 showed that more than $50 \%$ of patients, lay caregivers and members of the public preferred to be cared for and to die at home [15]. However, the quality of the 18 studies examined was low and the percentage of people who preferred home varied greatly, from $25 \%$ to $100 \%$. A methodological review in 2009 highlighted differences in methods of eliciting and

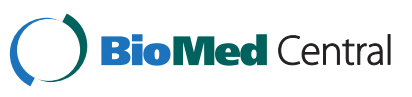


reporting preferences [16], but there may be other reasons why a preference for home is high in some studies and low in others. It is commonly accepted that dying in hospital remains frequent because people change their mind as their illness progresses, based in two studies conducted in the 1980s in London, UK $[17,18]$.

We aimed to examine the heterogeneity in estimates of a preference for home death. We explored reasons for variation, particularly in relation to the quality of studies, the way in which preferences were assessed and, for the first time, examined changes of preference with illness progression.

\section{Methods}

\section{Design}

Systematic review. The PRISMA checklist is available online in Additional file 1.

\section{Search strategy}

Three strategies were used to systematically identify relevant studies. First, in May 2011 we searched four databases - MEDLINE (1966-2011), EMBASE (19802011), psycINFO (1967-2011) and CINAHL (1982-2011) using a combination of MESH headings and 22 keywords (details online in Additional file 2). Secondly, we handsearched six palliative care journals January 2006 - June 2011 (Palliative Medicine, Journal of Palliative Medicine, Supportive Care in Cancer, Journal of Pain and Symptom Management, BMC Palliative Care, Journal of Palliative Care) to identify recent articles not available through databases. Thirdly, the references of 18 literature reviews and all included papers were tracked (references available from reviewers). Additional data on included studies were obtained via personal contact.

\section{Selection criteria}

Inclusion criteria were: original data on people's preferences for place of end of life care and/or place of death; a scenario (real or hypothetical) of advanced or severe stages of progressive disease (cancer or non-malignant); adult population; use of quantitative and/or qualitative methodologies (qualitative data aimed to complement areas with low or inconclusive quantitative evidence).

Exclusion criteria were: no examination of preferences but only factual place of care or place of death; preferences not explicitly for place of care at the end of life or place of death; preferences not framed in a scenario of advanced or severe stages of a progressive disease; children only; reviews, comments, case stories, historical, ethical or educational analysis or unpublished material; papers not written in English, French, German, Italian, Portuguese or Spanish (due to translation limits).

\section{Data extraction}

The data were extracted to a standard form and SPSS datasheets under the following headings: study type (quantitative, qualitative or using both methods); study design (retrospective, cross-sectional, prospective - depending on the assessment timing of preferences); population to which preferences referred to (general public, patients, lay caregivers, older people, health care professionals, medical or nursing students); country of origin; publication year; data collection year(s); setting (including whether the study was population-based or service-based); sample (including sample size, \% with cancer and \% in terminal stage for patient populations, i.e. with advanced/severe illness or who died before or during the study period); response rate; preferences assessment method (including which questions were asked and whether preferences referred to place of care or place of death); results (including percentage expressing a preference for home, changes over time and qualitative findings). BG or NC extracted the data from the papers and a second reviewer assessed a $10 \%$ sample of papers to check accuracy. Disagreements were resolved by consensus.

\section{Assessment of quality and strength of evidence}

The quality of individual studies was assessed using two different standardized scales for quantitative and qualitative research $([19,20]$, details in Additional file 3). Studies using both quantitative and qualitative methods were evaluated using both scales. Studies were classified as high, medium or low quality using final scores (high $\geq 70 \%$, medium $60 \%-69 \%$, low $<60 \%$ ). However, because final quality scores for quantitative and qualitative research reflect different criteria, direct comparisons between the two are not appropriate. BG or NC assessed the quality of studies; $\mathrm{SH}$ independently assessed a $7 \%$ sample of studies using quantitative methods and MG assessed a $50 \%$ sample of studies using qualitative methods (we checked a higher percentage of qualitative studies as initial comparisons showed more disagreement). Disagreements were resolved by consensus.

We graded the strength of the evidence adapting an algorithm from a review on factors associated with death at home for cancer patients [21]. This made use of the key elements for grading systems recommended by the US Agency for Healthcare Research and Quality - quality, quantity and consistency of the evidence [22]. We took high strength evidence from a minimum of three high quality studies in which $\geq 70 \%$ reported similar findings. Moderate strength evidence was measured amongst medium and high quality studies and it was present if there was a minimum of three high quality studies in which $<70 \%$ but $>50 \%$ reported similar findings or if $>50 \%$ of all studies reported similar findings with a minimum of three medium quality studies. If none of these 
requirements were met, the evidence was considered of low strength. The evidence was found inconclusive if the consistency was $50 \%$ (e.g. two studies showing different results) or when there was only one study. We graded the strength of evidence from all studies and of sub-groups: studies prior to 2000 and since 2000, studies in each population group, and studies examining changes of preference over time.

\section{Data synthesis}

We described the included studies in terms of country of origin, populations, design, assessment of preferences (outlining which questions were asked, when available) and year of publication (prior to and since 2000). We used charts to summarize the results of the quality assessment, separately for quantitative and qualitative research. Percentages of participants expressing a preference for dying at home in each study were calculated or extracted from the papers if numbers were not given. Studies that did not provide enough information to extract or calculate the percentage of participants with a preference for dying at home were excluded from further quantitative synthesis. Heterogeneity precluded the calculation of a summary measure. Instead, we examined ranges in percentages and numbers of studies where $>50 \%$ and $>70 \%$ of the participants expressed a home preference. These were analyzed by population group and the strength of the evidence was determined. We tabulated the methods and results of high quality studies.

Percentages of participants with a home preference were plotted separately for members of the general public, patients and caregivers (groups with more than ten studies), and according to study quality (high, medium, low). Percentages were weighted by sample size and ordered by year of publication to identify patterns. In studies measuring preferences for both place of care and place of death, we plotted place of death preferences; these were deemed most relevant given the policy and trends of increasing home deaths. For longitudinal studies with multiple measurements of preferences we plotted the average. When ideal and realistic preferences were assessed and reported we plotted the realistic preferences only. Cases with missing data were excluded from calculations.

For studies conducted with patients, we distinguished those where the majority had cancer. Country of origin, whether the question referred to place of care or place of death, study quality and the questions used to assess preferences were explored as reasons for heterogeneity. A narrative summary of the findings in each of the three population groups is provided, discussing outlying studies, defined as those with extreme estimates and small samples $(<100)$. We then analyzed changes in preferences over time, grading the strength of the evidence. We examined qualitative research on areas with low or inconclusive quantitative evidence, and integrated a narrative summary of the findings in the respective results section. Given the large number of studies included, we reference in the text only high quality studies included in the main analysis, outliers and key studies but all references are available from the reviewers.

\section{Results}

Searches, data extraction and disagreement between reviewers

Electronic searches yielded 839 references (PRISMA flowchart in Figure 1). Through scrutiny of abstracts, almost a third appeared eligible for inclusion; after examination of full versions, 140 were included. Handsearches, tracking of reference lists and spontaneous provision of studies by the authors added 100 papers. A total of 240 papers were reviewed, reporting 210 different studies: three papers presented two different datasets in which preferences were assessed differently in two populations (considered different studies), one presented four different datasets, and 36 reported on data from already included studies (these were merged with the first report). There were disagreements between the reviewers on the quality classification of 12 studies (three on quantitative methods and nine on qualitative methods).

\section{General overview}

The studies reported on the preferences for place of end of life care and/or place of death of 29,926 members of the general public, 34,021 patients, 19,514 caregivers, 11,613 older people, 3,504 health professionals (for their patients or themselves) and 1,729 medical or nursing students, from 33 countries.

Twenty-eight percent of the studies $(n=59)$ presented evidence from the US and $41 \%(n=86)$ were conducted in 17 countries in Europe (Belgium, Denmark, England, France, Germany, Hungary, Ireland, Israel, Italy, Netherlands, Norway, Scotland, Spain, Sweden, Switzerland, Turkey and Wales). Other countries included Australia, Canada, Hong Kong, Japan, New Zealand, Singapore, South Korea, Taiwan, and seven African countries (Ethiopia, Ghana, Rwanda, South Africa, Tanzania, Uganda and Zimbabwe). One qualitative study (ethnographic) explored preferences for place of death in the US and Japan, and another qualitative study examined the preferences of young adults with cancer in the UK, Germany and Australia (based on written narratives from their parents); an online survey from the British Medical Journal was UK-based but had worldwide coverage.

Nearly three quarters of the studies $(n=151)$ focused on patients or caregivers. In 73 studies most patients had cancer whilst in 37 studies most had non-malignant diseases such as dementia, human immunodeficiency virus (HIV)/ 


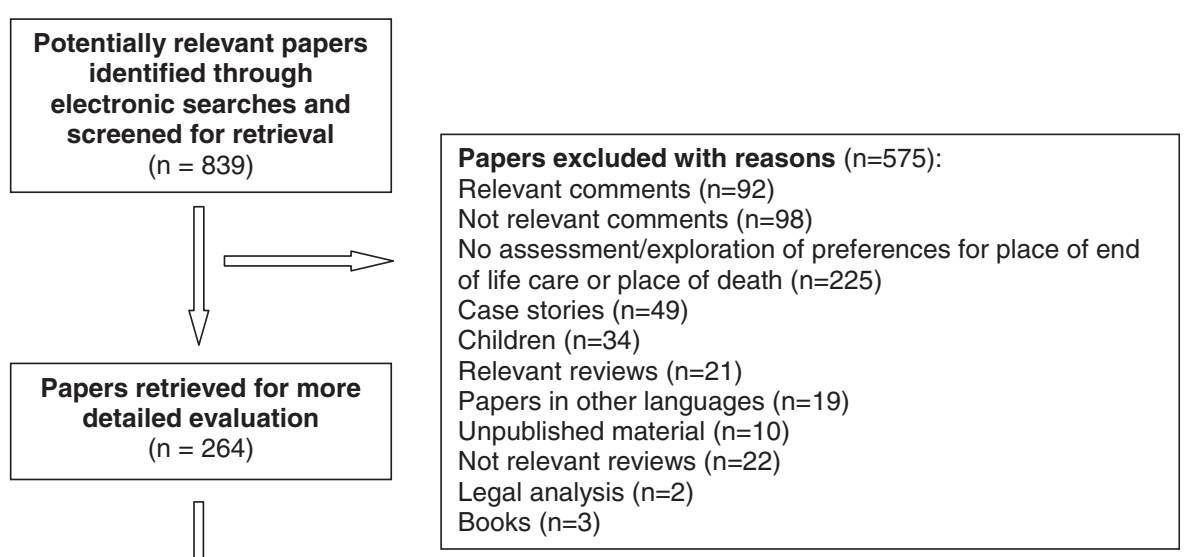

Papers excluded with reasons $(n=124)$ :

No assessment/exploration of preferences for place of end

of life care or place of death $(n=100)$

Relevant comments $(n=12)$

Not relevant comments $(n=2)$

Case stories $(n=6)$

Unpublished material $(n=3)$

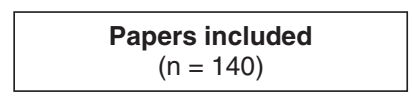

Unreachable $(n=1)$

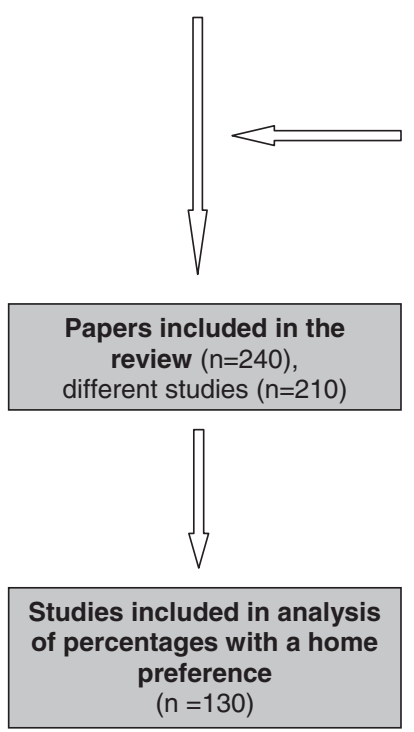

\begin{tabular}{|l|}
\hline Papers identified through handsearches $(n=28)$ \\
\hline $\begin{array}{l}\text { Papers identified through follow up of reference lists } \\
(n=67):\end{array}$ \\
Tacking reference lists of relevant reviews $(n=20)$ \\
Tracking reference lists of included papers $(n=47)$ \\
\hline Papers spontaneously provided by authors $(n=5)$ \\
\hline
\end{tabular}

review $(n=240)$

different studies $(n=210)$

Figure 1 Flow of studies through review.

acquired immunodeficiency syndrome (AIDS), heart failure, chronic obstructive pulmonary disease (COPD) and motor neurone disease (MND). In four studies there were equal proportions of patients with cancer and non-malignant conditions, and in the remaining 37 the percentage with cancer was unknown. The patients were deemed terminally ill in 129 studies.

\section{Progression of studies, methodological quality and assessment of preferences}

Only 34 out of the 210 studies were of high quality (16\%), 51 were medium quality (24\%) and 125 were low quality studies (60\%). Four of the 49 studies published prior to 2000 were considered of high quality. Since 2000, 161 new studies were published, 30 of high quality (19\%). Nearly three quarters of all studies used quantitative methods ( $\mathrm{n}=153$ ), 41 used qualitative methods and 16 made use of both (the qualitative component was dominant in four of these).

Around two thirds of the quantitative research was of low quality ( $n=114 / 169)$. Fourteen studies used quantitative methods at high standard. The inclusion/exclusion criteria for participants were generally clear and the assessment of preferences objective and reliable (Figure 2). 

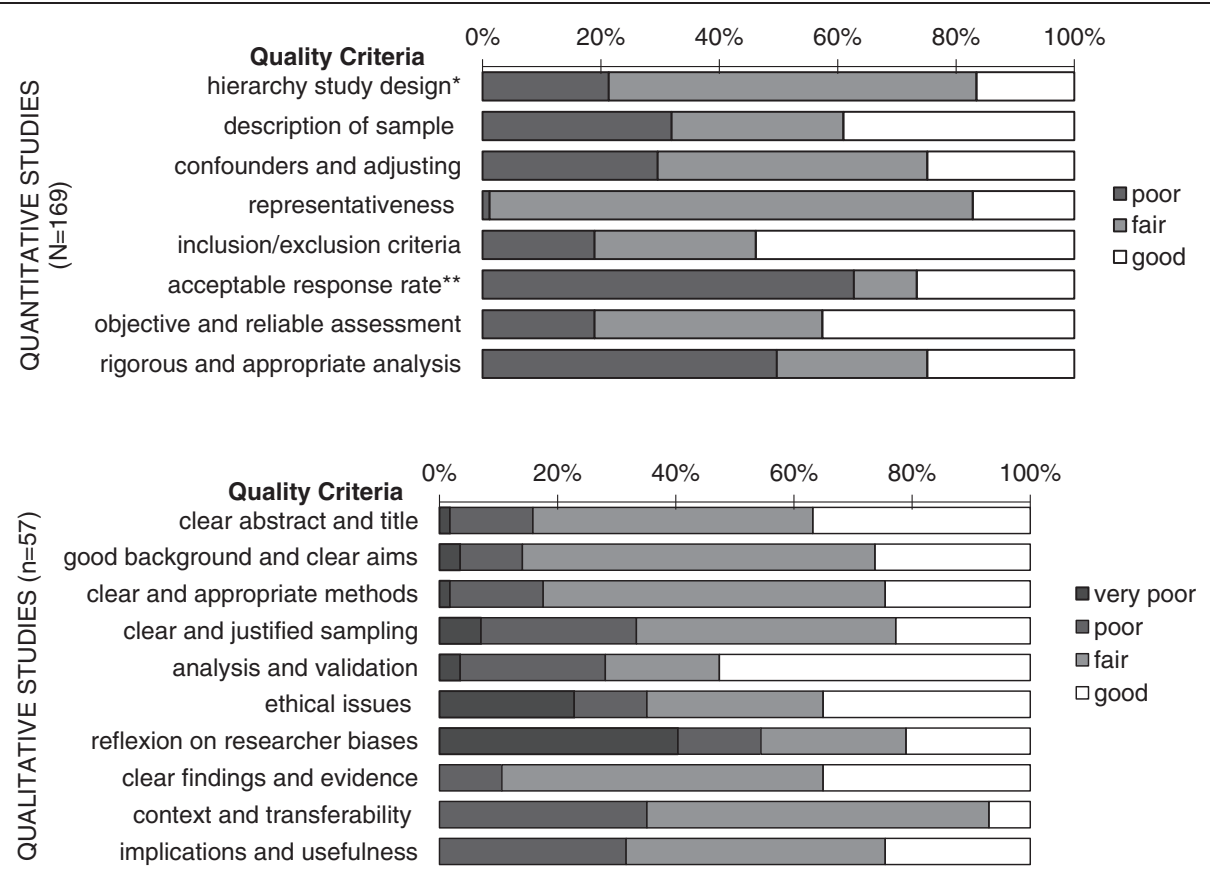

Figure 2 Quality assessment for studies using quantitative and qualitative methods. Footnote: Quantitative criteria applied to 169 studies (153 purely quantitative, 16 using both quantitative and qualitative methods). Qualitative criteria applied to 57 studies (41 purely qualitative and 16 using both quantitative and qualitative methods). ${ }^{*}$ Rating for study design (poor $=$ retrospective, fair $=$ cross-sectional, good $=$ prospective). ** Rating for acceptable response rate (poor $<60 \%$ or unknown, fair $60 \%-69 \%$, good $\geq 70 \%$ ).

Main weaknesses were poor response rates, inadequate design, potential for confounding, and limited analysis. Most were cross-sectional, assessing preferences at one point in time, 36 did this retrospectively and 15 studies provided data on preferences over time. Response rates were either below $60 \%$ or unknown in around three quarters of the studies $(\mathrm{n}=124)$.

More than a third (39\%) of the qualitative research was considered of high quality $(n=22 / 57)$. Almost all studies had a good background, using appropriate and specific qualitative methods and sampling techniques with clear aims. Lack of reflection on researcher biases, validation in the analysis and ethical issues were weaker points. The samples were often small (27 studies had $<40$ participants), which limited the transferability of the findings (found to be good in four studies only).

Seventy-six studies provided the exact question used for assessing preferences (details in Additional file 4). The questions were varied, with some enquiring directly if home was the preferred place, whilst others asked more broadly what the preferred place was or would be. In addition, 31 studies did not state the question but either described it briefly or referred to the tool from where the question was taken, and 103 were not fully clear on the terminology/context used, or did not mention the question or tool used at all.

\section{Preference for dying at home}

The percentage of participants who expressed a preference for dying at home was reported in 130 studies; 95 were published since 2000. Most examined preferences for place of death $(n=76), 41$ referred to place of care and ten to both place of care and place of death (three were unclear). Only 14 studies were of high quality, all but three published since 2000 (available online in Additional file 5). The 14 high quality studies report on preferences for 6,463 people $(1,400$ patients, 836 caregivers and 4,227 members of the general public) (Table 1).

We found moderate strength evidence that the majority of people preferred dying at home (Table 1). In 75\% of the studies $(97 / 130)>50 \%$ of participants expressed a preference for home (ranging from 51\% to 100\%). However, the consistency of findings amongst high quality studies was below 70\% (9/14), hence the evidence did not reach high strength. In around one third of all studies $(47 / 130)$ the percentage of participants expressing a preference for home was higher than $70 \%$.

Two high quality studies published prior to 2000 found that most participants preferred home - 59\% amongst 462 members of the general public in Adelaide and three rural areas in Australia and 73\% amongst 120 hospitalized AIDS patients in five Seattle tertiary care hospitals in the US $[23,24]$. Another high quality study, a 
Table 1 Preferences for dying at home: quantity, quality, consistency and strength

\begin{tabular}{|c|c|c|c|c|c|c|c|}
\hline \multicolumn{2}{|c|}{ Population group and studies } & \multicolumn{4}{|c|}{ High strength evidence (only high quality studies) } & \multicolumn{2}{|c|}{$\begin{array}{l}\text { Moderate evidence (high anc } \\
\text { medium quality studies) }\end{array}$} \\
\hline Population group & Number of studies & $\begin{array}{l}\text { Number of } \\
\text { participants }\end{array}$ & Consistency $^{a}$ & $\begin{array}{l}\text { Preference for } \\
\text { dying at home }\end{array}$ & $\begin{array}{l}\text { High } \\
\text { strength? }\end{array}$ & $\begin{array}{l}\text { Preference for } \\
\text { dying at home }\end{array}$ & $\begin{array}{l}\text { Moderate } \\
\text { strength? }\end{array}$ \\
\hline \multicolumn{8}{|c|}{ Evidence for $>50 \%$ preference for dying at home } \\
\hline All people & 130 & 6463 & $64 \%(9 / 14)$ & $>50 \%$ & no & $>50 \%$ & yes \\
\hline Patients & 92 & 1400 & $60 \%(6 / 10)$ & $>50 \%$ & no & $>50 \%$ & yes \\
\hline Caregivers & 36 & 836 & $60 \%(3 / 5)$ & $>50 \%$ & no & $>50 \%$ & yes \\
\hline General public & 26 & 4227 & $75 \%(3 / 4)$ & $>50 \%$ & yes & $\mathrm{n} / \mathrm{a}$ & $\mathrm{n} / \mathrm{a}$ \\
\hline Health professionals and students & 7 & 0 & $0 \%(0 / 0)$ & $>50 \%$ & no & $>50 \%$ & yes \\
\hline Older people & 9 & 0 & $0 \%(0 / 0)$ & $>50 \%$ & no & $>50 \%$ & no \\
\hline \multicolumn{8}{|c|}{ Evidence for $>70 \%$ preferences for dying at home (or $<70 \%$ ) } \\
\hline All people & 130 & 6463 & $29 \%(4 / 14)$ & $>70 \%$ & no & $<70 \%$ & no \\
\hline Patients & 92 & 1400 & $50 \%(5 / 10)$ & $>70 \%$ & no & $<70 \%$ & no \\
\hline Caregivers & 36 & 836 & $80 \%(4 / 5)$ & $<70 \%$ & yes & $\mathrm{n} / \mathrm{a}$ & $\mathrm{n} / \mathrm{a}$ \\
\hline General public & 26 & 4227 & $75 \%(3 / 4)$ & $<70 \%$ & yes & $\mathrm{n} / \mathrm{a}$ & $\mathrm{n} / \mathrm{a}$ \\
\hline Health professionals and students & 7 & 0 & $0 \%(0 / 0)$ & $>70 \%$ & no & $>70 \%$ & yes \\
\hline Older people & 9 & 0 & $0 \%(0 / 0)$ & $>70 \%$ & no & $>70 \%$ & no \\
\hline
\end{tabular}

Footnote: The table shows the total number of studies that reported a $>50 \%$ and a $>70 \%$ (or $<70 \%$ ) preference for dying at home amongst all types of participants and within each of five population groups. It then shows whether there is high strength evidence amongst all people and within each population group. High strength evidence was measured amongst only high quality studies and it was present, according to our grading system, if $\geq 70 \%$ of studies reported similar findings (e.g. there was no high strength evidence that $>50 \%$ of all people preferred dying at home because the consistency was $64 \%$, i.e. nine out of 14 studies showed estimates higher than $50 \%$ but five did not). The last two columns show whether there is moderate strength evidence; this was measured amongst medium and high quality studies and it was present, according to our grading system, if there was a minimum of three high quality studies in which $<70 \%$ but $>50 \%$ reported similar findings or if $>50 \%$ of all studies reported similar findings with a minimum of three medium quality studies (e.g. there was moderate strength evidence that $>50 \%$ of all people preferred dying at home because the consistency amongst high quality studies was $73 \%$, i.e. more than half of the studies showed estimates higher than $50 \%)$.

${ }^{a}$ Consistency of findings across studies is shown as a percentage (number of high quality studies pointing in same direction / total number of high quality studies on topic).

longitudinal study of 77 home care patients with cancer and their caregivers at a hospice in London (UK), showed that averaging preferences at different points in time, $77 \%$ of the patients and $59 \%$ of the caregivers preferred home [18]. However, 12 other high quality studies were published since 2000, increasing the diversity in populations, study designs, and in the phrasing of the questions used to assess preferences. The findings became more heterogeneous (with the percentage of people who preferred home ranging $5 \%$ to $100 \%$ ). As a result, the strength of evidence decreased from high to moderate.

\section{Heterogeneity between and within population groups}

The evidence within different population groups largely mirrored the general findings. Plots of percentages amongst the main groups - general public, patients and caregivers - confirmed that $>50 \%$ or more preferred dying at home across studies in all three groups (Figure 3). However, the plots also revealed wide heterogeneity between studies, particularly amongst those with patients (and to a lesser extent caregivers), when compared with the general public.
Once study quality was taken into account, evidence that the majority prefer dying at home graded moderate with two exceptions (Table 1). The evidence on general public was found to be strong, with three of four high quality studies from Australia and Spain showing that most of the general public preferred home [23,25-27]. The evidence on older people was found to have low strength. There were no high quality studies reporting the percentage of older people who preferred dying at home. A medium quality study in Quebec (Canada) found that $34 \%$ of 138 people aged 55 or over recruited via senior organizations preferred home [28]. Eight low quality studies reporting percentages from $39 \%$ to $94 \%$, the latter in a small-scale study with 49 people aged 60 or over recruited via newsletters for seniors, also in Canada [29].

Qualitative research filled the gap of high quality quantitative information on older people, with five studies exploring at length the preferences of this group in Canada, Ghana and the UK [29-33]. The findings showed that older people wished they would not have to move from their home. However, there were fears of burdening the family and requiring full-time nursing care due to dependency from a chronic limiting illness or while 


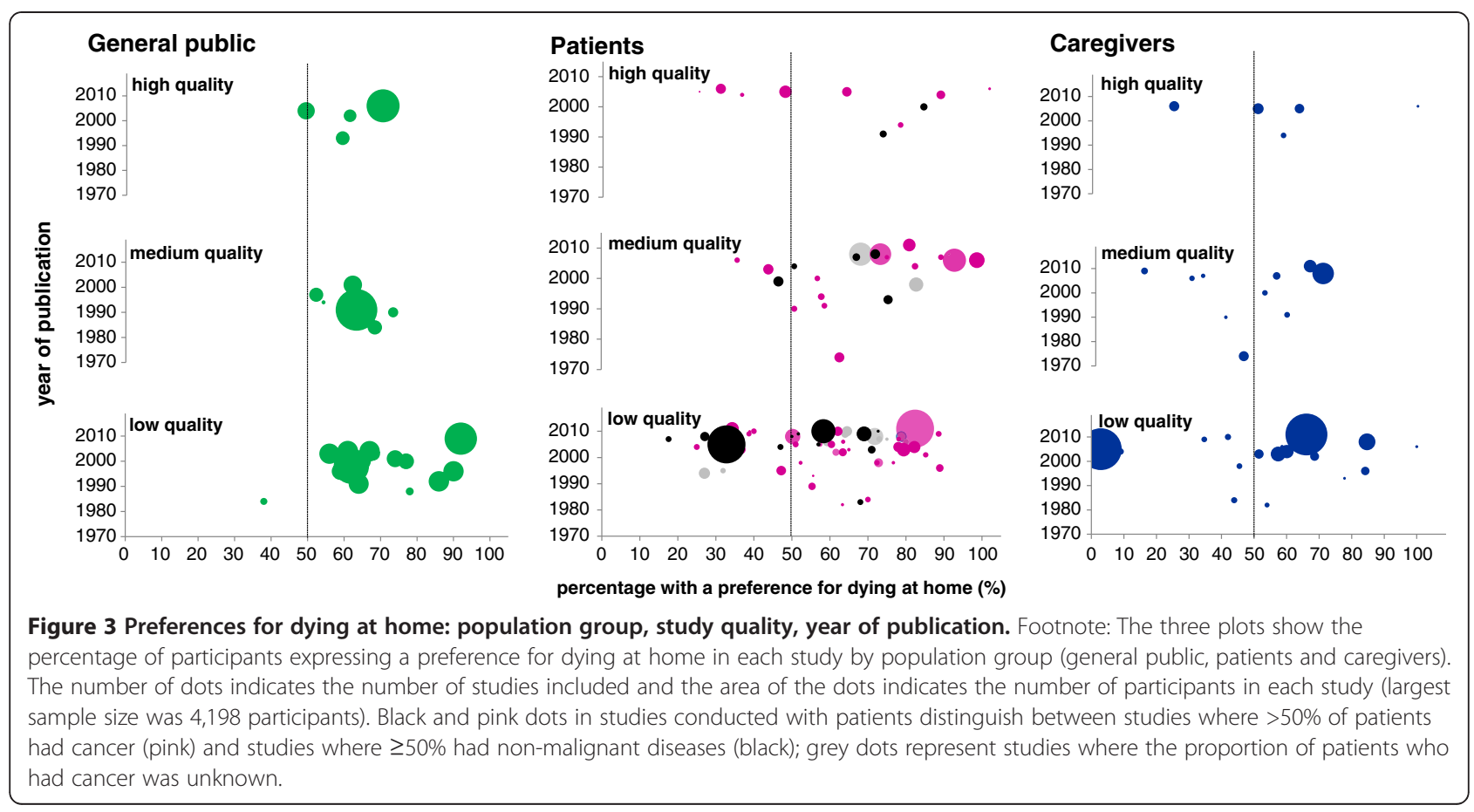

dying. Normative and moral values (i.e. cultural and religious beliefs involving death at home), and practical issues also played a role for older people (e.g. family witnessing suffering and having to manage pain and the dying body within the domestic space, concerns with the quality of care at home, presence of professionals in the home, involvement of children in intimate care, poor material conditions at home).

The preferences of the three main population groups are now examined in more detail.

\section{General public}

Twenty-four studies showed that the majority of the general public (52\% to $92 \%$ ) preferred dying at home. This was contradicted by two studies; one recruited 122 participants from local churches and a waiting room in a primary care center (US) of which $38 \%$ said they would choose to die at home if they "had a life-threatening illness" [34], and a study (high quality) conducted with 725 attendees in three health centers in Gipuzkoa (Spain), where $49 \%$ said that if they had cancer in terminal stage they would prefer to spend the last days at home [27]. Among the four high quality studies, estimates of a home preference ranged from $49 \%$ in Spain to $70 \%$ in Australia [23,25-27].

Three US studies (two nationwide telephone surveys conducted in the 1990s by market and social research companies for the National Hospice Organization, and one household survey in South Dakota) reported notably high percentages (86\%, 90\% and 92\%) [35-37]. Influenced by these studies, the US findings showed more variation than the eight surveys in Europe, which reported home preferences in Ireland (67\%), Italy $(62 \%, 64 \%)$, Spain (49\%, $59 \%, 61 \%)$ and the UK $(56 \%, 63 \%)$. A telephone survey conducted in two counties in Georgia (US) in 1990, found that although $73 \%$ preferred to die at home, $70 \%$ would like to be cared for in hospital in the prospect of being very sick [38]. There appeared to be no time trends, as shown in Figure 3.

\section{Patients and caregivers}

Overall, 94 studies determined the prevalence of a preference for dying at home amongst patients $(n=58$ studies), their caregivers $(n=2)$, or both $(n=34)$. Nearly half were conducted in the UK $(n=29)$ or the US $(n=17)$, but 21 other countries were also covered. In 54 studies, $>50 \%$ of the patients had cancer and in 23 studies the majority had other diseases (in 17 studies the percentage with cancer was unknown). In 82 studies the patients were deemed terminally ill.

Sixty-six studies out of 92 examining patient preferences found that most wished dying at home but there was wide variation (percentages ranging $18 \%$ to $100 \%$, Figure 3). Amongst the ten high quality studies and excluding two small-scale outliers in each extreme, estimates of a home preference ranged $31 \%$ to $87 \%$. Twenty-six studies from 13 countries found that $<50 \%$ of patients preferred home - in the UK (7 studies in England and 1 in Scotland), the US (4 studies), Spain (3 studies), Japan (2 studies), South Korea (2 studies), and Australia, Ethiopia, Rwanda, Sweden, Taiwan, Uganda, and Zimbabwe (with 1 study each). The lowest 
percentage (18\%) was found by a Spanish study regarding the preferences for place of death of 102 patients with congestive heart failure or end-stage dementia who died in two acute care hospitals, based on caregiver accounts one month after the patient died [39]. The highest percentages in studies where most patients had non-malignant conditions (89\% and $82 \%$ ) were lower than in studies where most patients had cancer $(100 \%$ and $92 \%)$.

Twenty-two out of 36 studies examining caregiver preferences found that the majority preferred their relatives to be at home, with less variation than in studies with patients (Figure 3). Estimates of a home preference ranged $25 \%$ to $64 \%$ amongst the five high quality studies, excluding one small-scale study at a community care hospice in Durham (US) where all caregivers preferred the patient to be cared for at home [40]. The lowest estimates were found in medium to low quality studies from Japan (3\%, 3\% and 15\%) and Ethiopia (9\%) [41-44].

Comparisons of home preferences for paired groups of patients and caregivers (provided by 34 studies, five of high quality) showed that the differences between the two groups were generally small - 17 studies found differences of $<10 \%$ (Figure 4 ). A home preference was more frequent amongst patients than amongst their caregivers in all but eight studies (two of high quality). However, statistical tests were rarely performed. Of the five high quality studies, two tested for differences and reported conflicting findings [45,46]; hence, the evidence on whether patient and caregiver's preferences for home differ was deemed inconclusive. A telephone survey of 216 bereaved relatives of patients who received home care in Ontario (96\% with cancer) [45] showed that relatives were more likely to prefer an institutional death ( $14 \%$ as compared to $5 \%$ of patients; $\mathrm{p}<0.001)$. In contrast, a study of 371 cancer patients and 281 caregivers in seven university hospitals and one national cancer centre in South Korea [46] found no differences regarding preferences for a home death (47\% patients and $51 \%$ caregivers, $\mathrm{p}=0.10$ ), although caregivers were less likely to prefer home as place of care compared to patients ( $49 \%$ vs. $53 \%$, respectively; $\mathrm{p}=0.02)$. The largest discrepancy was found in a Japanese study with nursing home residents with middle or advanced stages of dementia and their caregivers, where $15 / 29$ patients $(52 \%)$ but only $1 / 31$ caregivers $(3 \%)$ preferred the patient to die at their own home [42].

This heterogeneity between and within groups was corroborated by qualitative research. The case of advanced dementia was examined in focus groups with 39 family members of severely impaired nursing home residents in Minnesota, US [47]. The findings revealed complex negotiations between family and patients about moving into a nursing home (often dictated by need rather than preference); the use of the patient's personal historical identity and previous wishes to guide decision-making in the absence of overt preferences; and difficulties for the family in balancing the patient and their own wishes. Studies conducted in Canada, Japan and Denmark, mainly about cancer patients, showed that in some situations a preference for home was dyadic and equally important to patients and caregivers [48,49]; the result of a 'mutual pact' or promise to the patient, sometimes made in the context of a hospital admission or involving reciprocate care [50,51]. Some caregivers expressed a sense of achievement when the patient died at home, whilst others felt lack of choice, guilt and sorrow, depending on the outcome [50,51]. In Morecambe Bay (UK), Thomas et al. studied the preferences of 41 patients with advanced cancer and found they were strongly influenced by an assessment of caregivers' capacity to care, irrespective of caregivers' expressed desire to do it [52]. In Örebro (Sweden), Sahlberg-Blom et al. interviewed bereaved relatives of 56 cancer patients and found that patient participation in end of life decisions, including on where to die, varied from self-determination and co-determination to delegation and non-participation, depending on context, patient's personality, social network, cultural values and the extent to which their wishes and those of the caregivers could be supported [53].

\section{Changes over time}

Overall, 15 studies (3 high quality) measured if preferences changed over time (one of the general public, six of patients, seven of both patients and caregivers and one including professionals, patients and caregivers). In 13 studies, the data were collected prospectively, either through repeated interviews or observations with participants $(\mathrm{n}=$ 8 ) or from patient records $(n=5)$. Two studies enquired retrospectively (e.g. "Have your thoughts changed in any way in the last few months/weeks?"). Twelve studies (two of high quality) tracked individual changes (nine studies of patients, two of caregivers and one of both). The evidence was inconclusive as only the study with members of the public reported the statistical significance of the observed changes. A summary of the findings follows.

\section{Group changes}

An experiment with 183 members of the general public recruited via choir and music associations in the Netherlands, assessed preferences prior and after showing the participants' stories in text or video reflecting a combination of attributes relevant to place of death, and compared with a direct method of value elicitation [54]. The authors found significant shifts after text and video stories were shown. Participants became more negative about choosing to die at home and more positive about 


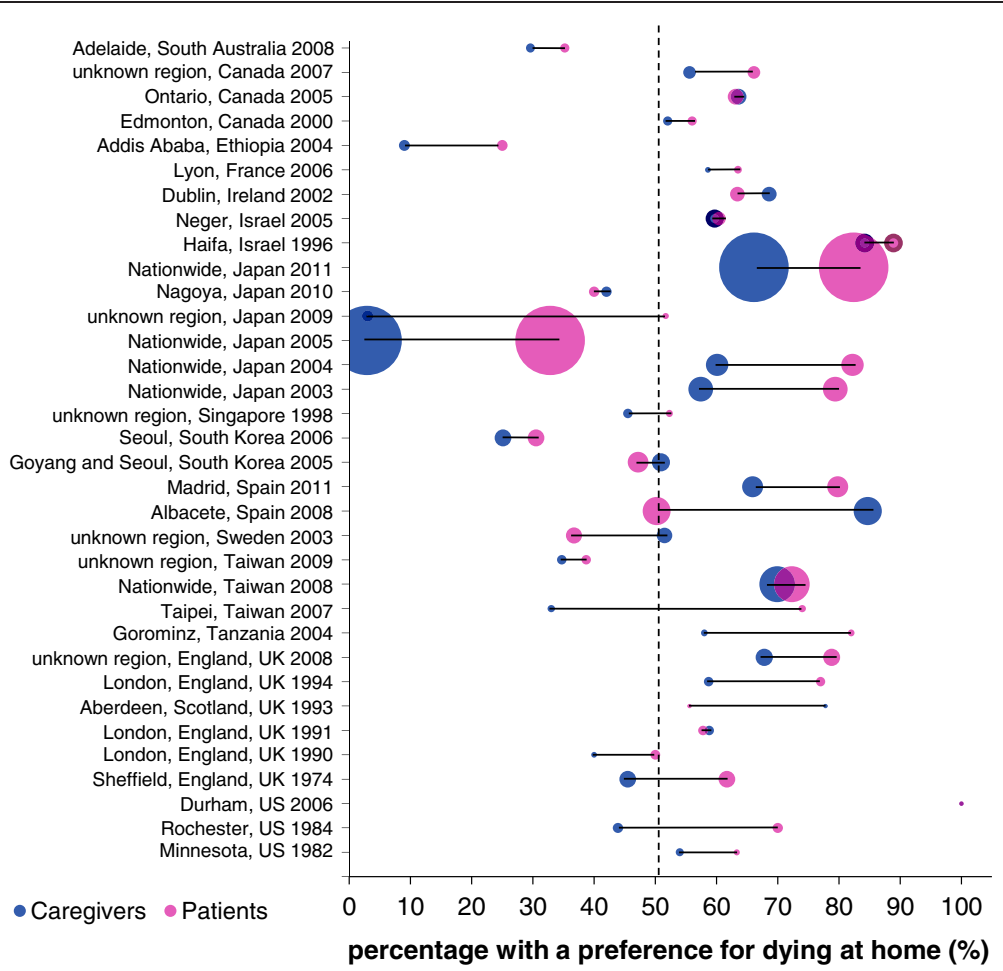

Figure 4 Preferences for dying at home amongst patients and their caregivers. Footnote: The plot shows in each study the percentage of patients and their caregivers expressing a preference for dying at home. The number of dots indicates the number of studies included ( $n=34$ ) and the area of the dots indicates the number of participants in the study (largest dot represents 4175 participants). Blue dots report to caregivers and pink dots to patients (dots are superimposed for one study as the percentages were 100\% for both patients and caregivers). Country region was unknown in six studies.

choosing to die in hospice, particularly older participants. Those who watched the video stories also became more positive towards nursing homes.

Townsend et al.'s study, conducted in 1986-87 in a London hospital, was the only study to comment on the significance of changes in patient preference over time [17]. The authors found a decline in home preferences in realistic circumstances (from 58\% to 50\%) and an increase in ideal circumstances (from $68 \%$ to $72 \%$ ), amongst 84 terminally ill patients. Townsend et al. stated that "the change in preference was not of a significant order", although it was unclear whether statistical tests were performed. Findings of three other studies may have reached statistical significance if tests were performed, of which two suggested declining preferences for home. A study with 125 older patients in a community-based call program in Baltimore (US) found that 99 patients changed their preferences for place of death, all but one from hospital to home [55]. Changes in the opposite direction were reported amongst home care patients at a London hospice in 1984-86 [18]. Preferences for home care decreased from $100 \%$ to $54 \%$ amongst 77 patients and from $100 \%$ to $45 \%$ amongst their caregivers in the last eight weeks before the patients died. A study embedded in the RCT of a palliative care program in Adelaide (Australia) was the most recent study to examine preference change through repeated interviews. This also reported decreases in a home preference, amongst 71 dyads of patients and caregivers [56]. Preferences for home as place of care fell from $87 \%$ to $71 \%$ amongst patients and $85 \%$ to $66 \%$ amongst caregivers, whilst a preference for home death fell from $41 \%$ to $35 \%$ amongst patients and from $42 \%$ to $30 \%$ amongst caregivers.

\section{Individual changes}

Changes in preference were documented for 277 of 1,395 patients (20\%) across ten studies (two of high quality). However, this ranged from $2 \%$ to $80 \%$. UK studies found changes for at least 31 of 243 palliative care patients in Yorkshire (with no evidence that changes were assessed for all) [57], four of 41 terminally ill cancer patients in Morecambe Bay (high quality study) [52], 5 of 21 terminally ill patients in a GP practice in Cambridge [58] approximately 61 of 166 patients seen by a hospital palliative care team in London [59], 5 of 298 patients in three hospices in the south east of England [60], four of 30 terminally ill cancer patients in 
a general practice in Scotland [61]. In addition, preferences changed for 99 of 125 older patients in the Baltimore community-based house call program (high quality study) [55]; 23 of 71 terminally ill patients in the Adelaide palliative care program [56], eight of 20 terminally ill patients who wanted to die in hospital in Ontario [62] and 37 of 380 cancer patients under the home palliative care team in Madrid [63]. The direction of changes varied but was most commonly from hospital to home, home to hospice and from home to hospital.

Three studies, none of high quality, found that less than a third of caregivers changed preference for the patient's place of death -21 of 71 caregivers of patients in the Adelaide palliative care program [56]; 3 of 18 caregivers of cancer patients at a hospital palliative care service in London [64] and 22 out of 205 caregivers (11\%) in Grande et al.'s study with patients referred to five Hospice at Home services in Cambridge (UK) [65].

\section{Qualitative research}

Eight studies conducted in Australia, Canada, Sweden, the UK and the US added qualitative information on changes over time, mostly presented in case stories [40,52,53,66-70]. Different patterns emerged, which resonated with the quantitative findings. Strong and consistent home preferences from patients and caregivers were reported, but so were cases of changes in preferences from home to institutions and from institutions to home. Health professionals in Australia and Canada said that patients and caregivers often change their minds $[69,70]$. Preferences changed from home to institutions due to uncontrolled pain and other symptoms ("she had terrible time breathing", incontinence, mental impairment), acute events (e.g. falls, injuries), treatment of reversible conditions for comfort and to maximize length of life, imminent death, caregivers burden/inability to safely care at home, increased need for care and dependency ("as I cannot go to the loo", "when he discovered he was incapable of being the host in his own home"), "naivety" on what to expect ("I've been watching too much TV, if I had known..."), and possible traumatic effects on children. Preferences changed from institutions to home due to the recognition that "prognosis was grim", limited beds available ("said she couldn't stay any longer"), unsatisfactory/uncomfortable hospital experiences (e.g. fighting for adequate pain control in hospital), and when assured of support from services at home.

\section{Differences between place of care and place of death}

Four studies (two of high quality) provided inconclusive evidence on whether patient preferences for place of care and for place of death differ. All four found that the majority preferred to be cared for at home and that fewer preferred to die at home, but only one carried out statistical tests. This study involved 380 patients with advanced cancer under a home palliative care team in Madrid (2004-06); 89\% preferred to be cared for at home and $80 \%$ preferred to die at home $(\mathrm{p}<0.001)$ [63]. Another study of 71 patients embedded in the RCT of the Adelaide palliative care programme (2002-04) found that more than two thirds preferred to be cared for at home $(87 \%$ when first assessed and $71 \%$ when last assessed) but only $40 \%-35 \%$ wanted to die at home [56]. No statistical tests were performed, though, and the same applied to two studies in South Korea, which found differences no greater than $6 \%[46,71]$.

Findings on caregivers were equally inconclusive with four studies showing contradictory results and statistical significance untested. The two South Korean studies suggested home was more frequently preferred as the place of death than the place of care amongst caregivers (but with differences no greater than 2\%) [46,71], whilst two studies - the Adelaide palliative care program RCT and Hinton's study of home care patients at a hospice in London (1984-86) - suggested the opposite [18,56].

Qualitative research showed distinctive conceptual features of preferences for place of care and for place of death $[40,50,72,73]$. A "definite" or "desperate" desire to remain at home as long as possible, with the ultimate hope to die at home or until admission if required, was viewed as dignifying if by the time the move happened the person was not aware of it anymore [50,72]. Perceptions of family's ability to care impacted on place of care preferences, whereas consequences of witnessing death at home (e.g. for children) impacted on place of death preferences $[50,73]$. Regardless of place of death, some surviving spouses said the value was frequently on the place where the majority of terminal care was provided and expressed satisfaction at having mastered the time spent at home $[40,50]$.

\section{Discussion}

In this systematic review we found numerous studies providing moderate evidence that the majority of people prefer dying at home (this was reported in $75 \%$ of 130 studies). We also found, based on 10 studies, that around four fifths of patients did not change preference as their illness progressed. There was, however, wide variation. This was observed even amongst high quality studies. Heterogeneity was lowest amongst general public studies and greatest amongst patient studies. We also found indications that a preference for home may be less frequent amongst lay caregivers and older people with qualitative research suggesting that although home is generally the ideal preference, circumstances may make this seem impossible. Qualitative research also revealed a conceptual distinction between preferring home as the place of care and as the place of death, although the 
evidence was inconclusive on whether there are statistically significant differences between the two.

\section{Majority prefers to be at home}

Our findings showed that this applies to different groups in the population - most importantly to patients (in 87\% of the studies they were deemed terminally ill). Having found consistent results observed by different researchers in different places with different samples strengthens the likelihood of the finding to be true [74]. Although the body of evidence identified presented important methodological weaknesses, such as lack of clarity regarding the questions used to elicit preferences, there are now more high quality studies than in 2000 [15]. Still, caution is needed due to the observed variation in prevalence estimates of a preference for home, even amongst high quality studies (ranging $31 \%$ to $87 \%$ amongst patients, excluding outliers). This highlights the need to take the heterogeneity seriously and explore whether it may reflect real diversity in preferences for dying at home.

\section{Heterogeneity}

Our review included a large number of studies with varied features and populations. Methodological differences (e.g. quality of studies) and clinical differences (e.g. population group) explained some of the variation in findings between studies, but not all. There was still considerable heterogeneity within population groups and when 'controlling' for quality (as shown in Figure 3). This suggests that although the majority prefers dying at home, there may be diversity in individual preferences. This conclusion, however, takes study-level variation as indicative of individual-level variation and thus carries the risk of an interpretation error ("ecological fallacy") [75].

Data on patients and caregivers from the same study allowed us to make more direct and robust comparisons. Although statistical significance was rarely tested (hence the evidence was inconclusive), in the majority of the studies a home preference was higher amongst patients than amongst their caregivers. This corroborated previous findings [15]. The difference, should it be statistically significant, is important as the care provided by caregivers and their preferences are strong factors associated with the likelihood of the patients to die at home [21]. Qualitative findings suggested that caregivers commit to providing care and to address the patient's preference to be at home, to then become aware of the complexities involved. This highlights the importance of good communication of preferences and concerns between patients and caregivers throughout the process, and the need for practical and emotional support to caregivers, to meet the patient's preference when possible and to minimize the risk of difficult bereavement for caregivers.

\section{Ideal and realistic preferences}

Public preferences for home do not appear to be higher than patients' and caregivers' but the evidence is stronger and more homogenous (Figure 3). This finding suggests there may be less consensus in the face of reality and, as Townsend et al. suggested, an important distinction between preferences in ideal and realistic circumstances [17]. Real life situations reported in qualitative research raised the issue of whether it is possible to prepare for acute events and to encourage things that have been found to keep ideal preferences possible (e.g. services at home, available and able caregivers). However, it is still not clear cut whether preferences for patients and caregivers change significantly over time. There is a real need for high quality studies on this matter.

\section{Limitations}

Our review has several limitations: search boundaries, the subjectivity introduced by the quality and grading criteria (although this involved independent reviewers and disagreement checks), and the reliance on data provided by relatively low quality quantitative research (although high and moderate strength evidence was only taken from high and medium quality studies). It is arguable that the quality assessment scales could have placed greater emphasis on how the data were collected and on the measurement of preferences, although this would give greater weight to a specific aspect of quality in detriment of others. Nevertheless, the way preferences are assessed is very important when analysing the findings. We urge consideration of any applicable measurement biases while interpreting the results of the different studies; to help with this we have provided the exact wording of the questions used to elicit preferences when this was known (Additional file 4). The review focused on a preference for home but we acknowledge the importance of other places for care and death, particularly hospices and palliative care units (studies suggest they are the second most frequent preference) [15] and care homes (increasingly important in ageing populations). Furthermore, the searches resulted in a large number of studies related to cancer patients (cancer was the only disease-specific search term used). A review targeted to non-malignant conditions such as COPD and advanced dementia may discover further literature for increasingly relevant groups for end of life care provision.

\section{Conclusions}

In this systematic review, we observed that the majority of people prefer dying at home. This is aligned with the direction taken by current end of life care strategies to target a home setting [11]. Notwithstanding, even in countries where these strategies exist, the majority of people still do not die at home $[3,14,76]$. This highlights the need for 
stronger action on factors previously found to influence death at home [21] so that more are able to have their preferences met. There is also the need for further research to understand what factors influence death at home for people dying from non-malignant conditions, where the evidence is thinner and the chances of dying at home are generally lower than for cancer patients $[14,76]$.

At the same time, our findings urge rigorous and regular monitoring of preferences, and consideration of the diversity in views, with attention to older people and caregivers. The strength of the evidence supporting that the majority prefers home to other settings is not as strong as it once was and there is a substantial minority of patients and caregivers for whom home is not the first choice or who change their mind. In face of an increasing demand of end of life care $[3,4,8,9]$, the findings highlight the importance of allowing for a degree of diversity and flexibility in service planning, alongside the focus on home care.

\section{Additional files}

\section{Additional file 1: PRISMA checklist. \\ Additional file 2: Search strategy.}

Additional file 3: Quality assessment criteria for quantitative and qualitative research.

Additional file 4: Questions used to assess preferences.

Additional file 5: High quality studies.

\section{Abbreviations}

AIDS: Acquired immunodeficiency syndrome; COPD: Chronic obstructive pulmonary disease; HIV: Human immunodeficiency virus; MND: Motor neurone disease; RCT: Randomized controlled trial; UK: United Kingdom; US: United States.

\section{Competing interests}

The authors declare that they have no competing interests.

\section{Authors' contributions}

BG and IJH conceived the idea and obtained funding; BG and NC conducted the searches, identified and retrieved the studies, extracted the data and assessed quality. SH conducted quality assessment for quantitative research and MG for qualitative research. All authors developed the methods, analyzed and interpreted the data, wrote the report and are guarantors. All authors read and approved the final manuscript.

\section{Acknowledgements}

We thank Cicely Saunders International for the financial support needed to undertake this systematic review including BG's PhD Research Training Fellowship, as part of a long-term research project on place of death led by BG and IJH http://www.cicelysaundersinternational.org/. The funders had no role in study design, data collection and analysis, decision to publish, or preparation of the manuscript. We also thank study authors who supplied additional information and Sam Hart and Fliss Murtagh for their help with the plots. Thanks to Professors Gunn Grande and Christine Ingleton, and to the reviewers for their insightful comments.

Received: 20 November 2012 Accepted: 11 February 2013 Published: 15 February 2013

\section{References}

1. Bowling A: The hospitalisation of death: should more people die at home? J Med Ethics 1983, 9(3):158-161.

2. Teno JM, Clarridge BR, Casey V, Welch LC, Wetle T, Shield R, Mor V: Family perspectives on end-of-life care at the last place of care. JAMA 2004, 291(1):88-93.

3. World Health Organization: Palliative Care for Older People: Best Practices. Copenhagen: World Health Organization; 2011.

4. Mathers CD, Loncar D: Projections of global mortality and burden of disease from 2002 to 2030. PLoS Med 2006, 3(11):e442.

5. Office for National Statistics: 2010-based National Population Projections; 2011. http://www.ons.gov.uk/ons/rel/npp/national-population-projections/ 2010-based-projections/index.html.

6. Population Division USCB: 2009 National Population Projections; 2009. http:// www.census.gov/population/projections/.

7. World Health Organization: Preventing chronic disease: a vital investment. Geneva: World Health Organization; 2005.

8. Gomes B, Higginson IJ: Where people die (1974-2030): past trends, future projections and implications for care. Palliat Med 2008, 22(1):33-41.

9. Gomes B, Cohen J, Deliens L, Higginson IJ: International trends in circumstances of death and dying amongst older people. In Living with ageing and dying: palliative care and end of life care for older people. Firstth edition. Edited by Gott M, Ingleton C. Oxford: Oxford University Press; 2011:3-18.

10. Houttekier D, Cohen J, Surkyn J, Deliens L: Study of recent and future trends in place of death in Belgium using death certificate data: a shift from hospitals to care homes. BMC Public Health 2011, 11:228.

11. Department of Health Human Services: Medicare and Medicaid Programs: hospice conditions of participation [final rule]. Fed Regist 2008, 73(109): 32088-32219.

12. Department of Health: End of Life Care Strategy - promoting high quality care for all adults at the end of life. London: Department of Health; 2008.

13. Flory J, Yinong YX, Gurol I, Levinsky N, Ash A, Emanuel E: Place of death: U. S. trends since 1980. Health Aff 2004, 23(3):194-200.

14. Gomes B, Calanzani N, Higginson IJ: Reversal of the British trends in place of death: time series analysis 2004-2010. Palliat Med 2012, 26(2):102-107.

15. Higginson IJ, Sen-Gupta GJA: Place of care in advanced cancer: a qualitative systematic literature review of patient preferences. $J$ Palliat Med 2000, 3(3):287-300.

16. Bell CL, Somogyi-Zalud E, Masaki KH: Methodological review: measured and reported congruence between preferred and actual place of death. Palliat Med 2009, 23(6):482-490.

17. Townsend J, Frank AO, Fermont D, Dyer S, Karran O, Walgrove A, Piper M: Terminal cancer care and patients' preference for place of death: a prospective study. BMJ 1990, 301(6749):415-417.

18. Hinton J: Can home care maintain an acceptable quality of life for patients with terminal cancer and their relatives? Palliat Med 1994, 8(3):183-196.

19. Khan KS: Undertaking systematic reviews of research on effectiveness: CRD's guidelines for those carrying out or commissioning reviews, Volume 2nd. York: University of York; 2001.

20. Hawker S, Payne S, Kerr C, Hardey M, Powell J: Appraising the evidence: reviewing disparate data systematically. Qual Health Res 2002, 12:1284-1299.

21. Gomes B, Higginson IJ: Factors influencing death at home in terminally ill patients with cancer: systematic review. BMJ 2006, 332:515-521.

22. West S, King V, Carey TS, Lohr KN, McKoy N, Sutton SF, Lux L: Systems to Rate the Strength of Scientific Evidence. Evidence Report/Technology Assessment No. 47 (Prepared by the Research Triangle Institute-University of North Carolina Evidence-based Practice Center under Contract No. 290-97 -0011). AHRQ Publication No. 02-E016. Rockville, MD: Agency for Healthcare Research and Quality; 2002.

23. Ashby M, Wakefield M: Attitudes to some aspects of death and dying, living wills and substituted health care decision-making in South Australia: public opinion survey for a parliamentary select committee. Palliat Med 1993, 7(4):273-282.

24. McCormick WC, Inui TS, Deyo RA, Wood RW: Long-term care preferences of hospitalized persons with AIDS. J Gen Intern Med 1991, 6(6):524-528.

25. Foreman LM, Hunt RW, Luke CG, Roder DM: Factors predictive of preferred place of death in the general population of South Australia. Palliat Med 2006, 20(4):447-453.

26. Fernández Suárez A, Fernandez AT, Alonso AC, Marcilla EM, Cortina VA, Conde RM, Rubio DJ: Attitudes of health centre users to diagnoses of terminal illness. Aten Primaria 2002, 30(7):449-454 
27. Rubio Arribas V, Martinez SE, Sarasola ZM, Benito Gl, Redin AS, Ibanez TV: [Cancer diagnosis: do we want to know the truth?]. [Spanish]. Aten Primaria 2004, 33(7):368-373.

28. Martineau I, Blondeau D, Godin G: Choosing a place of death: the influence of pain and of attitude toward death. J App/ Soc Psychol 2003, 33(9):1973-1993.

29. Wilson DM: End-of-life care preferences of Canadian senior citizens with caregiving experience. J Adv Nurs 2000, 31(6):1416-1421.

30. van der Geest S: Dying peacefully: considering good death and bad death in Kwahu-Tafo, Ghana. Soc Sci Med 2004, 58(5):899-911.

31. Gott M, Seymour J, Bellamy G, Clark D, Ahmedzai S: Older people's views about home as a place of care at the end of life. Palliat Med 2004, 18(5):460-467.

32. Seymour J, Payne S, Chapman A, Holloway M: Hospice or home? Expectations of end-of-life care among white and Chinese older people in the UK. Sociol Health IIIn 2007, 29(6):872-890.

33. Lloyd-Williams M, Kennedy V, Sixsmith A, Sixsmith J: The end of life: a qualitative study of the perceptions of people over the age of 80 on issues surrounding death and dying. J Pain Symptom Manage 2007, 34(1):60-66.

34. Smith RA, Wallston BS, Wallston KA, Forsberg PR, King JE: Measuring desire for control of health care processes. J Pers Soc Psychol 1984, 47(2):415-426.

35. National Hospice and Palliative Care Organisation: Press release: Spread the News: National Hospice and Palliative Care Organization. Alexandria: National Hospice and Palliative Care Organization; 1992

36. National Hospice and Palliative Care Organization: Press relase: New Findings Address Escalating End-of-Life Debate. Alexandria: National Hospice and Palliative Care Organization; 1996

37. Schrader SL, Nelson ML, Eidsness LM: "South Dakota's dying to know": a statewide survey about end of life. J Palliat Med 2009, 12(8):695-705.

38. Neubauer BJ, Hamilton CL: Racial differences in attitudes toward hospice care. Hosp J 1990, 6(1):37-48

39. Formiga F, Olmedo C, Lopez-Soto A, Navarro M, Culla A, Pujol R: Dying in hospital of terminal heart failure or severe dementia: the circumstances associated with death and the opinions of caregivers. Palliat Med 2007, 21(1):35-40

40. Evans WG, Cutson TM, Steinhauser KE, Tulsky JA: Is there no place like home? Caregivers recall reasons for and experience upon transfer from home hospice to inpatient facilities. J Palliat Med 2006, 9(1):100-110.

41. Shinoda-Tagawa T, Ikegami N: Resident and facility characteristics associated with the site of death among Japanese nursing home residents. Age Ageing 2005, 34(5):515-518

42. Nakanishi M, Honda T: Processes of decision making and end-of-life care for patients with dementia in group homes in Japan. Arch Gerontol Geriatr 2009, 48(3):296-299.

43. Miyashita M, Morita T, Ichikawa T, Sato K, Shima Y, Uchitomi Y: Quality indicators of end-of-life cancer care from the bereaved family members perspective in Japan. J Pain Symptom Manage 2009, 37(6):1019-1026.

44. World Health Organization: A Community Health Approach to Palliative Care for HIV/AIDS and Cancer Patients. Geneva: World Health Organization; 2004

45. Brazil K, Howell D, Bedard M, Krueger P, Heidebrecht C: Preferences for place of care and place of death among informal caregivers of the terminally ill. Palliat Med 2005, 19(6):492-499.

46. Choi KS, Chae YM, Lee CG, Kim SY, Lee SW, Heo DS, Kim JS, Lee KS, Hong YS, Yun YH: Factors influencing preferences for place of terminal care and of death among cancer patients and their families in Korea. Support Care Cancer 2005, 13(8):565-572.

47. Elliott BA, Gessert CE, Peden-McAlpine C: Family decision-making in advanced dementia: narrative and ethics. Scand J Caring Sci 2009, 23(2):251-258.

48. Thomson C, Brazil K: Insights on family decision making regarding place of care and death: bereavement interviews with family caregivers. . 17th International Congress on Palliative Care, September 23-26, 2008/ Palais Des Congres, Montreal, Canada. J Palliat Care 2008, 24(3):211-211.

49. Hirai K, Miyashita M, Morita T, Sanjo M, Uchitomi Y: Good death in Japanese cancer care: a qualitative study. J Pain Symptom Manage 2006, 31(2):140-147

50. Fisker T, Strandmark M: Experiences of surviving spouse of terminally ill spouse: a phenomenological study of an altruistic perspective. Scand J Caring Sci 2007, 21(2):274-281.

51. Clemmer SJ, Ward-Griffin C, Forbes D: Family members providing home-based palliative care to older adults: the enactment of multiple roles. Can J Aging 2008, 27(3):267-283.
52. Thomas C, Morris SM, Clark D: Place of death: preferences among cancer patients and their carers. Soc Sci Med 2004, 58(12):2431-2444.

53. Sahlberg-Blom E, Ternestedt B, Johansson J: Patient participation in decision making at the end of life as seen by a close relative. Nurs Ethics 2000, 7(4):296-313

54. Kerstholt $\mathrm{JH}$, Bart $\mathrm{H}$, Cremers $\mathrm{A}$ : Construction of health preferences: $a$ comparison of direct value assessment and personal narratives. Med Decis Making 2009, 29(4):513-520.

55. Leff B, Kaffenbarger KP, Remsburg R: Prevalence, effectiveness, and predictors of planning the place of death among older persons followed in community-based long term care. J Am Geriatr Soc 2000, 48(8):943-948.

56. Agar M, Currow DC, Shelby-James TM, Plummer J, Sanderson C, Abernethy AP: Preference for place of care and place of death in palliative care: are these different questions? Palliat Med 2008, 22(7):787-795.

57. Daley A, Sinclair K: Recording and auditing preferred place of death. Palliat Med 2006, 20(6):637-638.

58. Bowers B, Roderick S, Arnold S: Improving integrated team working to support people to die in the place of their choice. Nurs Times 2010, 106(32):14-16.

59. Gerrard R, Campbell J, Minton O, Moback B, Skinner C, McGowan C, Stone $P C$ : Achieving the preferred place of care for hospitalized patients at the end of life. Palliat Med 2011, 25(4):333-336.

60. Holdsworth L, Fisher S: A retrospective analysis of preferred and actual place of death for hospice patients. Int J Palliat Nurs 2010, 16(9):424-430.

61. Carroll DS: An audit of place of death of cancer patients in a semi-rural Scottish practice. Palliat Med 1998, 12(1):51-53.

62. McWhinney IR, Bass MJ, Orr V: Factors associated with location of death (home or hospital) of patients referred to a palliative care team. CMAJ 1995, 152(3):361-367.

63. Alonso-Babarro A, Bruera E, Varela-Cerdeira M, Boya-Cristia MJ, Madero R, Torres-Vigil I, De Castro J, Gonzalez-Baron M: Can this patient be discharged home? Factors associated with at-home death among patients with cancer. J Clin Oncol 2011, 29(9):1159-1167.

64. Higginson IJ, Hall S, Koffman J, Riley J, Gomes B: Time to get it right: are preferences for place of death more stable than we think? Palliat Med 2010, 24(3):352-353.

65. Grande GE, Ewing G, National Forum for Hospice at Home: Informal carer bereavement outcome: relation to quality of end of life support and achievement of preferred place of death. Palliat Med 2009, 23(3):248-256.

66. Scannell-Desch EA: Prebereavement and postbereavement struggles and triumphs of midlife widows. J Hosp Palliat Nurs 2005, 7(1):15-22.

67. Sorbye LW: Homecare at the end of life: a study of fifteen patients. Scand J Caring Sci 1990, 4(3):107-113.

68. McCall K, Rice AM: What influences decisions around the place of care for terminally ill cancer patients? Int J Palliat Nurs 2005, 11(10):541-547.

69. Clayton JM, Butow PN, Arnold RM, Tattersall MH: Discussing end-of-life issues with terminally ill cancer patients and their carers: a qualitative study. Support Care Cancer 2005, 13(8):589-599.

70. Stajduhar Kl, Davies B: Variations in and factors influencing family members' decisions for palliative home care. Palliat Med 2005, 19(1):21-32.

71. Yun YH, You CH, Lee JS, Park SM, Lee KS, Lee CG, Kim S: Understanding disparities in aggressive care preferences between patients with terminal illness and their family members. J Pain Symptom Manage 2006, 31(6):513-521.

72. Aoun S, Kristjanson $\sqcup$, Oldham L, Currow D: A qualitative investigation of the palliative care needs of terminally ill people who live alone. Collegian 2008, 15(1):3-9.

73. Riley J, Fenton G: A terminal diagnosis: the carers' perspective. Couns Psychother Res 2007, 7(2):86-91.

74. Hill AB: The environment or disease: association or causation. Proc $R$ SoC Med 1965, 58:295-300.

75. Robinson WS: Ecological correlations and the behavior of individuals. J Am Stat Assoc 1935, 30:517-536.

76. Cohen J, Houttekier D, Onwuteaka-Philipsen B, Miccinesi G, Addington-Hall J, Kaasa S, Bilsen J, Deliens L: Which patients with cancer die at home? A study of six European countries using death certificate data. J Clin Oncol 2010, 28(13): 2267-73.

doi:10.1186/1472-684X-12-7

Cite this article as: Gomes et al:: Heterogeneity and changes in preferences for dying at home: a systematic review. BMC Palliative Care $201312: 7$. 